\title{
Intellectual disability-strabismus syndrome
}

INSERM

\section{Source}

INSERM. (1999). Orphanet: an online rare disease and orphan drug data base. Intellectual disability-strabismus syndrome. ORPHA:363528

Intellectual disability-strabismus syndrome is a rare, genetic, syndromic intellectual disability disorder characterized by moderate to severe intellectual disability and esotropia. Other associated features may include growth failure (underweight, failure to thrive, short stature), microcephaly, tone abnormalities (hypotonia, spasticity), epilepsy, behavioral problems (hyperactivity, aggressiveness), and/or abnormal brain morphology, including arachnoid cyst, cerebral atrophy, mild ventriculomegaly, abnormal CNS myelination or corpus callosum agenesis. 